\title{
EDITORIAL
}

\section{Sleep apnoea and cancer: current insights and future perspectives}

\author{
Miguel Ángel Martínez-García*,\#, Francisco Campos-Rodríguez and Ramon Farré ${ }^{\#,+}$
}

I t seems paradoxical to observe how obstructive sleep apnoea (OSA) has been associated with a large number of systemic diseases, and is even recognized as a risk factor for some of them [1-4], but is nevertheless, strictly speaking, basically a local mechanical problem resulting from intermittent collapses of the upper airway during sleep. The explanation for this phenomenon is that these repetitive airway obstructions trigger several pathophysiological mechanisms that have an overall systemic effect. Thus, sympathetic activation, periodical hypoxaemias-normoxaemias, hypercapnia, arousals and changes in intrathoracic pressure induced by OSA lead to the activation of some pathophysiological pathways similar to those observed in several cardiovascular diseases [5]. This explains why OSA has been associated with the onset, poor control and worsened prognosis of some of the most prevalent cardiovascular diseases, such as arterial hypertension [6], cardiac arrhythmias [7], and cerebral and cardiovascular events [3, 4]. One aspect that has been far less studied to date is the relationship between OSA and systemic inflammation. In this respect, OSA has recently been associated with diseases with a great inflammatory burden, such as psoriasis [8] and other autoimmune disorders [9]. The relative scientific youthfulness of OSA means that new action mechanisms and previously unknown associations with other diseases are now being increasingly discovered. One particular piece of news was sprung on the scientific community at recent international congresses, creating quite a stir: some studies in both animals [10-12] and humans [13-15], undertaken almost simultaneously, had found an association between OSA and cancer.

There have been reports of a relationship between different aspects of nonapnoeic sleep disorders, particularly sleep duration, and cancer. Specifically, some studies have shown an association between short sleep duration and increased incidence of malignant tumours, particularly breast cancer [16, 17], although these findings have not been replicated by other authors [18]. Several mechanisms have been postulated to explain this association, most notably the increased production of pro-inflammatory cytokines and the suppression of melatonin

\footnotetext{
*Servicio de Neumología, Hospital Universitario y Politécnico La Fe, Valencia, ${ }^{\#}$ CIBER de Enfermedades Respiratorias, Bunyola, "Servicio de Neumología, Hospital Universitario de Valme, Seville, and +Unitat de Biofisica i Bioenginyeria, Facultat de Medicina, Universitat de BarcelonaIDIBAPS, Barcelona, Spain.

CORRESPONDENCE: M.Á. Martínez-García, Servicio de Neumología, Hospital Universitario y Politécnico La Fe, Bulevar Sur s/n, 46013 Valencia, Spain. E-mail: mianmartinezgarcia@gmail.com
}

secretion, which have been shown to inhibit cancer development and growth [16, 19, 20]. Another association has been reported between long sleep duration [21] or long daytime naps [22] and higher incidence of cancer. It has been postulated that this association could be explained by intermediate variables such as a poorer underlying state of health in people given to prolonged siestas [16, 22]. Nevertheless, until very recently, there was no literature available on a possible relationship between OSA and cancer, even though both diseases share some common pathophysiological mechanisms. However, it is interesting to note that there was already speculation about a possible relationship between these two diseases, in which intermittent hypoxia would play a fundamental role, back in 2007 [23].

Intermittent hypoxia is one of the most specific landmarks of OSA and has proven to induce considerable consequences at both tissue and systemic levels [24]. It has been reported that intermittent hypoxia plays an important role in regulating the different stages of tumour formation and progression [25]. In a situation of cellular hypoxia (typically found in tumours), the adaptive response is orchestrated by a family of transcription factors, the most important and most fully studied probably being hypoxia-inducible factor (HIF)-1. HIF-1 activates the transcription of genes that play critical roles in angiogenesis, the oxidative stress response, genetic instability, immune evasion, metabolic reprogramming, invasion and metastasis, radiation resistance, and stem-cell maintenance related to cancer [26]. Among all the effects of HIF-1, two stand out as being especially relevant. On the one hand, the overexpression of HIF-1 has been associated with a sharp increase in reactive oxygen species generated during reoxygenation periods, which can also play an important role in modifying gene expression by regulating the activity of some transcription factors. These include activator protein- 1 , which is known to play a pivotal role in tumourigenesis, and nuclear factor- $\kappa \mathrm{B}$, which has been associated with tumour development via the induction of apoptotic inhibitor factors, proliferation molecules, matrix metalloproteases and pro-angiogenic factors $[25,27]$. On the other hand, overexpression of HIF-1 has also been associated with some angiogenic molecules, of which the most important is probably vascular endothelial growth factor (VEGF). Angiogenesis has been shown to play a crucial role in both the growth of primary tumours and their metastasis. Tumour vessels boosted by VEGF are structurally and functionally abnormal, and even highly vascularised cancers may contain areas of severe hypoxia and necrosis. Both 
abnormal vascularisation and necrosis have been associated with the progression and poor prognosis of cancer [28].

On the basis of this background, which suggests a plausible association between intermittent hypoxia and a greater incidence and extension of tumours, a recent study in an animal model tested this pathophysiological hypothesis. The authors showed that when mice were subjected to an intermittent hypoxia pattern mimicking OSA, the growth rate of melanoma tumours was two-fold greater than in normoxic control animals [10]. Moreover, the mice subjected to intermittent hypoxia presented a greater number of pulmonary metastases [11].

These promising findings in experimental research encouraged other groups with available longitudinal data on large sleep cohorts to assess a potential link between OSA and cancer in humans. Two large-scale series, one population-based and one clinically based, have very recently studied this possible relationship, simultaneously but independently of each other. First, an association between OSA and cancer has been reported in a population-based cohort from Wisconsin (USA) $(1,522$ subjects; 22-yr follow-up) [13]. The results substantiated a significant increase in the probability of death from cancer in patients with severe OSA. Meanwhile, the other database research projects involved two parallel studies from the Spanish Sleep Network involving $\sim 5,000$ patients with a median follow-up of nearly 5 yrs. These studies have reported an increased cancer incidence [15] and mortality [14] in OSA patients exhibiting nocturnal arterial oxygen desaturations as compared with those who did not experience nocturnal hypoxaemia even when the results were adjusted by the most important confounding factors (age, sex, smoking, alcohol consumption and body mass index). Moreover, the association between OSA and cancer proved to be more intense in younger subjects (aged $<65 \mathrm{yrs}$ ) and in males. Interestingly, this is partly consistent with some reports on the relationship between OSA and cardiovascular disease [3, 4]. Remarkably, in both the Wisconsin and Spanish cohort studies, the association was stronger when the hypoxaemia index was used as a marker of the severity of the disease and when patients with good adherence to continuous positive airway pressure (CPAP) treatment were excluded from the analysis, opening up the possibility of a protective effect derived from CPAP (although this parameter was not the main outcome and, accordingly, the results presented less statistical power and must be interpreted with caution). Therefore, the findings from both the experimental and clinical data carried out to date point in the same direction: towards a genuine association between OSA and cancer, in which intermittent hypoxia would play a key role.

However, this newly reported association raises many more questions than answers. These open issues must be addressed in coming years, given their considerable potential importance in epidemiological, prognostic and therapeutic terms, particularly bearing in mind that OSA is a potentially treatable disease. One obvious key aspect is to corroborate the results of the aforementioned recent studies through the analysis of other large databases. Another important pending task is to carry out large-scale prospective studies with prolonged follow-up in both patients with OSA (to evaluate the incidence of cancer) and in patients with cancer (to evaluate tumour progression or response to treatment according to OSA severity).

The search for causality in the OSA-cancer relationship will be particularly complicated, as it will be very difficult to trace the onset of the natural history of the two diseases. Moreover, data in patients with well-established cancer will need to be controlled for a large number of confounding factors, especially those related to the various antitumoural treatments currently in use. Another key factor will be the identification of the pathophysiological pathways that would best explain the association between OSA and cancer. Two nonexclusive mechanisms seem to be of especial relevance. On the one hand, the presumed carcinogenic role of the biological insults (e.g. intermittent hypoxaemia or sleep disruption) associated with recurrent sleep apnoeas, which would trigger increased cancer incidence. On the other hand, the role of these biological insults in a faster extension and deteriorated prognosis of a pre-existing cancer. It will also be important to ascertain whether the OSA-cancer association varies in accordance with the location or histological type of the tumour, since different types of malignant cells have different adaptive responses to intermittent hypoxia.

The identification of confounding variables will also be crucial. Any investigation of the relationship between OSA and cancer clearly has to take into account confounders, the most important probably being (apart from the well-known ones such as smoking, alcohol consumption and age) obesity and sleep duration. Obesity (and, by extension, metabolic syndrome) has proved to be a disease involving a low-intensity chronic inflammatory component, altering the balance of oxidative stress and modifying many hormones, growth factors and other mediators potentially contributing to the development and progression of cancer-related processes [12, 28]. Moreover, obesity is capable of generating a certain degree of chronic hypoxia, especially at night, and this is also associated with a greater carcinogenic capacity [26]. Therefore, the hypothesis that the relationship between obesity and cancer could involve the presence of OSA as an intermediate explanatory variable cannot be ruled out. Another confounding variable that must be taken into account, on the basis of the current literature, is the quantity and quality of patient sleep, which, as mentioned above, is also associated with a higher incidence of cancer. It is also important to identify the polysomnographic indices that best describe this association so far the most viable option seems to focus on hypoxaemia indices - and the threshold values associated with an increased risk of cancer. Finally, an essential question that merits further evaluation, providing this association between OSA and cancer is confirmed, is the potential role of CPAP treatment, more specifically whether the effect of anti-tumour drugs is modified by OSA treatment with CPAP.

The possible relationship between OSA and cancer reveals an excellent gateway to further research and an unbeatable opportunity to undertake multinational studies that would answer the very many questions that still remain with respect to this relationship. Meanwhile, however, caution is required because the recent promising findings are, at the moment, merely a gleam of light in a door that still remains closed, although all the information available to date seems to indicate where we need to push to finally open it. 


\section{SUPPORT STATEMENT}

This work was supported, in part, by the Spanish Ministry of Economy and Competitiveness (SAF2011-22576) and SEPAR (2010 and 058/2011).

\section{STATEMENT OF INTEREST}

None declared.

\section{REFERENCES}

1 Marín JM, Carrizo SJ, Vicente E, et al. Long-term cardiovascular outcomes in men with obstructive sleep apnoea-hypopnoea with or without treatment with continuous positive airway pressure: an observational study. Lancet 2005; 365: 1046-1053.

2 Peppard PE, Young T, Palta M, et al. Prospective study of the association between sleep-disordered breathing and hypertension. N Eng J Med 2000; 342: 1378-1384.

3 Redline S, Yenokyan G, Gottlieb DJ, et al. Obstructive sleep apneahypopnea and incident stroke: the Sleep Heart Health Study. Am J Respir Crit Care Med 2010; 182: 269-277.

4 Gottlieb DJ, Yenokyan G, Newman AB, et al. Prospective study of obstructive sleep apnea and incident coronary heart disease and heart failure: the Sleep Heart Health Study. Circulation 2010; 122: 352-360.

5 Shamsuzzaman AS, Gersh BJ, Somers VK. Obstructive sleep apnea: implications for cardiac and vascular disease. JAMA 2003, 290: 1906-1914.

6 Goodfriend TL, Calhoun DA. Resistant hypertension, obesity, sleep apnea, and aldosterone: theory and therapy. Hypertension 2004; 43: 518-524.

7 Mehra R, Benjamin EJ, Shahar E, et al. Association of nocturnal arrhythmias with sleep-disordered breathing. Am J Respir Crit Care Med 2006; 173: 910-916.

8 Yang YW, Kang JH, Lin HC. Increased risk of psoriasis following obstructive sleep apnea:a longitudinal population-based study. Sleep Med. 2012; 13: 285-289.

9 Kang JH, Lin HC. Obstructive sleep apnea and the risk of autoinmune diseases: a longitudinal population-based study. Sleep Med 2012; 13: 583-588.

10 Almendros I, Montserrat JM, Ramírez J, et al. Intermittent hypoxia enhances cancer progression in a mouse model of sleep apnoea. Eur Respir J 2012; 39: 215-216.

11 Almendros I, Montserrat JM, Torres M, et al. Intermittent hypoxia increases melanoma metastasis to the lung in a mouse model of sleep apnea. Eur Respir J 2012; 40: Suppl. 56, 8s.

12 Almendros I, Montserrat JM, Torres M, et al. Obesity and intermittent hypoxia increase tumor growth in a mouse model of sleep apnea. Sleep Med 2012; [Epub ahead of print DOI: 10.1016/ j.sleep.2012.08.0120].
13 Nieto FJ, Peppard P, Young T, et al. Sleep disordered breathing and cancer mortality: Results from the Wisconsin Sleep Cohort Study. Am J Respir Crit Care Med 2012; 186: 190-194.

14 Martínez-García MA, Campos-Rodríguez F, Duran J, et al. Association between sleep apnea and cancer mortality. Longitudinal multicenter study in 5,467 patients from the Spanish Cohort. Eur Respir J 2012; 40: Suppl. 56, 709s.

15 Martínez-García MA, Martínez M, Durán-Cantolla J, et al. Association between sleep apnea and cancer incidence. Longitudinal study of 8900 patients from the multicenter Spanish Cohort. Am J Respir Crit Care Med 2012; 185: A6723.

16 von Ruesten A, Weikert C, Fierze I, et al. Association of sleep duration with chronic diseases in the European Prospective Investigation into Cancer and Nutrition (EPIC)-Postdam Study. PLOS ONE 2012; 7: e30972.

17 Kanikazi M, Kuriyama S, Sone T, et al. Sleep duration and the risk of breast cancer: the Ohsaki Cohort Study. Br J Cancer 2008; 99: 1502-1505.

18 Piñeiro SP, Schemhammer ES, Tworoger SS, et al. A prospective study on habitual duration of sleep and incidence of breast cancer in a large cohort of women. Cancer Res 2006; 66: 5521-5525.

19 Wu AH, Wang R, Koh WP, et al. Sleep duration, melatonin and breast cancer among Chinese woman in Singapore. Carcinogenesis 2008; 129: 1244-1248.

20 Blask DE. Melatonin, sleep disturbance and cancer risk. Sleep Med Rev 2009; 13: 257-264.

21 McElroy JA, Newcomb PA, Titus-Ernstoff L, et al. Duration of sleep and breast cancer risk in a large population-based casecontrol study. J Sleep Res 2006; 15: 241-249.

22 Cairns BJ, Travis RC, Wang XS, et al. A short-time increase in cancer risk associated with daytime napping is likely to reflect preclinical disease: prospective cohort study. Br J Cancer 2012; 107: $527-530$

23 Abrams B. Cancer and sleep apnea - the hypoxia connection. Med Hypotheses 2007; 68: 232.

24 Levy P, Pépin JL, Arnau C, et al. Intermittent hypoxia and sleepdisordered breathing: current concepts and perspectives. Eur Respir J 2008; 32: 1082-1095.

25 Toffoli S, Michiels C. Intermittent hypoxia is a key regulator of cancer cell and endothelial cell interplay in tumours. FEBS J 2008; 275: 2991-3002.

26 Semenza GL. Oxigen sensing, homeostasis and disease. N Engl J Med 2011; 365: 537-547.

27 Reuter S, Gupta SC, Chaturvedi MM. Oxidative stress, inflammation, and cancer: how are they linked? Free Radic Biol Med 2010; 49 1603-1616.

28 Hursting SD, Hursting MJ. Growth signals, inflammation, and vascular perturbations. Mechanistic links between obesity, metabolic syndrome and cancer. Arterioscler Thromb Vasc Biol 2012; 32: 1766-1770. 